\title{
CONSUMO DE MORERA (Morus alba) Y SORGO NEGRO FORRAJERO (Sorghum almum) EN GANADO JERSEY 1
}

\author{
Carlos Boschini ${ }^{2}$
}

\section{RESUMEN}

Consumo de morera (Morus alba) y sorgo negro forrajero (Sorghum almum) en ganado Jersey. Se cosecharon ramas de morera de 84 días y forraje de sorgo negro forrajero de 77 días de rebrote. El forraje fue suministrado a seis toretes Jersey con un peso vivo promedio de $337( \pm 24,27) \mathrm{kg}$, distribuidos en un diseño de cuadrado latino 3 x 3 , repetido. Los tratamientos experimentales fueron: a.- suministro único de morera, b.- $50 \%$ de morera y $50 \%$ de sorgo negro forrajero en base verde tal como fue cosechada y c.- suministro único de sorgo negro forrajero. Ambos forrajes se ofrecieron a los animales en forma picada (hojas y tallos). La mezcla de morera y sorgo se realizó en partes iguales en base al peso fresco. Cada animal recibió $50 \mathrm{~kg} /$ día de forraje verde. La relación promedio de hoja:tallo en la morera fue de $1,3( \pm 0,39)$ y de 0,84 $( \pm 0,26)$ en el sorgo negro. Se recolectaron muestras del material ofrecido y rechazado para el análisis de materia seca, proteína cruda, fibra neutro detergente y cenizas totales. No se observaron diferencias $(p>0,05)$ en el consumo de materia fresca y de materia seca ( $\mathrm{kg} / \mathrm{animal} / \mathrm{dí}$ ) entre los tratamientos. La ingesta de materia seca por cada $100 \mathrm{~kg}$ de peso vivo de los animales fue de $1,94 \%$ para la dieta de morera, $1,82 \%$ para la mezcla morera/sorgo y $1,67 \%$ para la dieta de sorgo negro, lo cual fue inferior al $2 \%$ esperado.

\begin{abstract}
Mulbeery (Morus alba) and black sorghum fodder (Sorghum almum) intake in Jersey cattle. Branches of mulberry and black sorghum fodder were harvested on the 84th and 77th day of growth respectively. Both kinds of forage were given to six Jersey steers, whose average weight was $337( \pm 24.27) \mathrm{kg}$. The animals were distributed according to a repeated Latin square $3 \times 3$. The experimental treatments were: a. only mulberry, b. half mulberry and half sorghum, and c. only black sorghum. All the forage was freshly chopped (leaves and stems) and measured in equal parts. Each animal received $50 \mathrm{~kg} /$ day of fresh forage. The average leaf:stem ratio was $1.3( \pm 0.39)$ in the mulberry and $.84( \pm 0.26)$ in the black sorghum. Samples were taken of the forage given in the morning and that which was leftover from the next day in order to analyze the dry material, crude protein, neutral dtergent fiber and ashes. No significant differences $(p>0.5)$ were observed in the fresh and dry matter intake (kg/animal/day) among the treatments. The dry matter intake for every $100 \mathrm{~kg}$ of body weight was $1.94 \%$ for the mulberry diet, $1.82 \%$ for the mulberry-sorghum mixture and $1.67 \%$ for the sorghum diet, all of which were less than the $2 \%$ expected.
\end{abstract}

\section{INTRODUCCIÓN}

La morera ha mostrado excelentes características de palatabilidad y en consecuencia un alto consumo por el ganado caprino (Benavides, et al., 1994; Ortiz, 1992; Castro, 1989). La ganadería bovina de leche enfrenta el reto de hacer más eficaz los alimentos forrajeros que consume, asi como mantener y superar los rendimientos actuales. Se espera que los modelos de alimentación futuros tengan en cuenta la sostenibilidad y el uso racional de los recursos naturales (Castro, et al., 1994). La morera, introducida en las dos últimas décadas como cultivo agrosilvoforrajero, se ha adaptado bien en las zonas de los altiplanos con alta luminosidad y de mane- ra excelente en las dos áreas tropicales de América Central (Benavides, et al., 1994; Rodríguez, et al., 1994; Boschini, et al., 1998). Se reporta que la planta entera tiene un contenido proteico que varía entre 14 a $22 \%$ de proteína cruda en la materia seca (Piccioni, 1970) y la digestibilidad in vitro va de 70 a $80 \%$ (Ortiz, 1992). Las muestras de morera provenientes de la zona alta de Cartago en la Meseta Central de Costa Rica, reportado por UFAG Laboratorien de Suiza, muestran valores de 22\% en proteína cruda, $19 \%$ de fibra cruda, $2,3 \%$ de extracto etéreo, $50 \%$ de proteína sobrepasante y un estimado de 1,48 Mcal/kg de energía neta para lactación, en base seca. Eswara y Reddy, 1992, evaluando el valor nutritivo de las hojas de morera en cabras y ovejas, reporta-

\footnotetext{
1 Inscrito en la Vicerrectoría de Investigación, No.737-98-318.

2 Estación Experimental Alfredo Volio Mata. Facultad de Agronomía. Universidad de Costa Rica.
} 
ron consumos de materia seca de 2,74 a $3,55 \%$ del peso vivo, con contenidos de $12 \%$ y $71 \%$ de proteína cruda y total de nutrimentos digeribles, respectivamente.

En Asia, el valor bromatológico de la morera radica especialmente en la hoja (Rao, et al.; 1996; Yen, et al., 1996, Subbarayappa, et al., 1995). En América Central, los estudios conducidos al aprovechamiento de este forraje en la ganadería, han sido orientados al aprovechamiento de la rama completa (hojas y tallos) (González, J.G., 1996; Estrada, A., 1995; Benavides et al., 1994; Ortiz, 1992; Castro, 1989; Rodríguez, et al., 1994; Oviedo, F.J., 1995; Boschini, et al. 1998). Espinoza, E. (1996) reporta valores medios de relación hoja:tallo de 0,57 a 0,99 tomados en tres zonas geográficas diferentes de Costa Rica. En la Meseta Central, Boschini et al., 1989, observaron relaciones hoja:tallo superiores a uno antes de los 100 días de crecimiento. Las proporciones varían de acuerdo a las condiciones ambientales y manejo de la explotación. Al ganado bovino, se le ofrece la planta entera de la morera, previamente picada. Lo anterior constituye una mezcla de hoja y tallos que el animal selecciona durante el consumo, tomando en cuenta que al menos un $50 \%$ del suministro constituyen tallos no comestibles. El presente trabajo tuvo el objetivo, de comparar la selección y consumo de morera y de una gramínea de corte como es el sorgo negro forrajero, empleado en la alimentación del ganado lechero con resultados satisfactorios.

\section{MATERIALES Y MÉTODOS}

El experimento se llevó a cabo en la Estación Experimental de Ganado Lechero "Alfredo Volio Mata" de la Universidad de Costa Rica, ubicada a $1542 \mathrm{msnm}$, con una precipitación anual media de $2050 \mathrm{~mm}$, distribuida durante los meses de mayo a noviembre. La temperatura media es de $19,5^{\circ} \mathrm{C}$ y la humedad relativa media de $84 \%$.

El forraje de morera fue extraído de un cultivo con tres años de edad, en explotación continua. Se programó una uniformización escalonada cronológicamente para que el forraje tuviese una edad de 84 días en el momento de la cosecha. El sorgo negro forrajero se extrajo de un cultivo de dos años, cosechado regularmente. Se le practicó una uniformización similar a la realizada con la morera , para lograr un forraje con 77 días de rebrote al cosecharse. Ambos cultivos recibieron una fertilización nitrogenada equivalente a $150 \mathrm{~kg} / \mathrm{ha} / \mathrm{año}$ de nitrógeno.

El forraje cosechado fue suministrado a seis toretes Jersey con un peso vivo promedio de $337( \pm 24,27) \mathrm{kg}$, distribuidos en un diseño de cuadrado latino 3 x 3 , repetido. Los tratamientos experimentales fueron: a. Suministro único de morera, b. $50 \%$ de morera y $50 \%$ de sorgo negro forrajero en base verde tal como fue cosechada y c. Suministro único de sorgo negro forrajero. Ambos forrajes se ofrecieron a los animales en forma picada $(2,5 \mathrm{~cm})$ tal como fueron cosechados (hojas y tallos). La mezcla del tratamiento de morera y sorgo se realizó en partes iguales en base al peso verde. Cada animal fue ubicado en un comedero individual, recibiendo diariamente $50 \mathrm{~kg}$ de forraje verde. La relación promedio de hoja:tallo en la morera fue de $1,3( \pm 0,39)$ y de $0,84( \pm 0,26)$ en el sorgo negro. Cada periodo experimental fue de 28 días y previamente, los animales tuvieron 14 días de adaptación a la nueva dieta. Las tres dietas fueron ofrecidas en dos porciones de $25 \mathrm{~kg}$ cada una, la primera a las 8:00 am y la segunda a las 4:00 pm. El forraje rechazado se pesó diariamente a las 7:00 am. En días alternos se recolectaron las muestras del material ofrecido y rechazado para su análisis de laboratorio. Los animales consumieron agua ad-libitum de un bebedero automático individual. No se suplieron sales minerales. En las muestras recogidas se analizó el contenido de materia seca, de proteína cruda, de fibra neutro detergente y de cenizas totales, mediante los procedimientos aprobados (AOCA, 1980; Goering y Van Soest, 1970) en cada caso.

Los datos observados se analizaron con el PROC GLM del paquete estadístico SAS (1985). Las fuentes de variación que resultaron estadísticamente, se sometieron a la prueba de Dunca para diferenciar la significancia entre medias.

\section{RESULTADOS Y DISCUSIÓN}

El consumo de materia fresca, materia seca, proteína cruda, fibra neutro detergente y cenizas totales de las dietas: morera, morera/sorgo y sorgo negro forrajero, se presenta en el Cuadro 1. No se observó diferencias ( $\mathrm{P}>0,05)$ en el consumo de materia fresca y de materia seca (kg/animal/día) entre los tratamientos, a pesar del mayor $(\mathrm{P}>0,05)$ contenido $(\%)$ de materia seca ofrecida en la dieta de morera respecto a las otras. El contenido de materia seca del forraje consumido fue similar ( $\mathrm{P}>0,05)$ en los tres tratamientos. El contenido de proteína cruda de la materia seca ofrecida en la dieta de morera fue superior $(\mathrm{P}>0,01)$ a la mezcla de morera/sorgo y esta similar a la dieta de sorgo negro. El contenido de proteína cruda en la materia seca consumida resultó diferente $(\mathrm{P}>0,01)$ en los tres tratamientos, decreciendo conforme disminuyó la cantidad de morera presente en la dieta. La concentración de fibra neutro 
detergente en la materia seca ofrecida y consumida fue notoria $(P>0,01)$ entre las tres dietas estudiadas. La fibra neutra se incrementó conforme se incluyó mayor cantidad de sorgo negro forrajero en la dieta. El contenido de cenizas totales en la materia ofrecida fue diferente $(P>0,01)$ en las tres dietas experimentales. Sin embargo, la concentración total de minerales en la materia seca consumida no mostró variaciones de importancia $(\mathrm{P}>0,05)$.

El consumo diario de proteína cruda, fibra y cenizas fue altamente diferenciado $(\mathrm{P}>0,01)$ en las tres dietas experimentales (Cuadro 1). Si bien, la cantidad de materia seca consumida diariamente no mostró variaciones estadísticas importantes ( $\mathrm{P}>0,05)$, en la Figura 1 se observa el comportamiento en el consumo de las cuatro fracciones estudiadas, observándose en todos los casos una clara tendencia lineal positiva para materia seca, proteína cruda y cenizas, conforme prevalece la morera sobre el sorgo negro en la dieta.

Al cuantificar el consumo de materia seca por cada $100 \mathrm{~kg}$ de peso vivo de los animales, se observa una re-

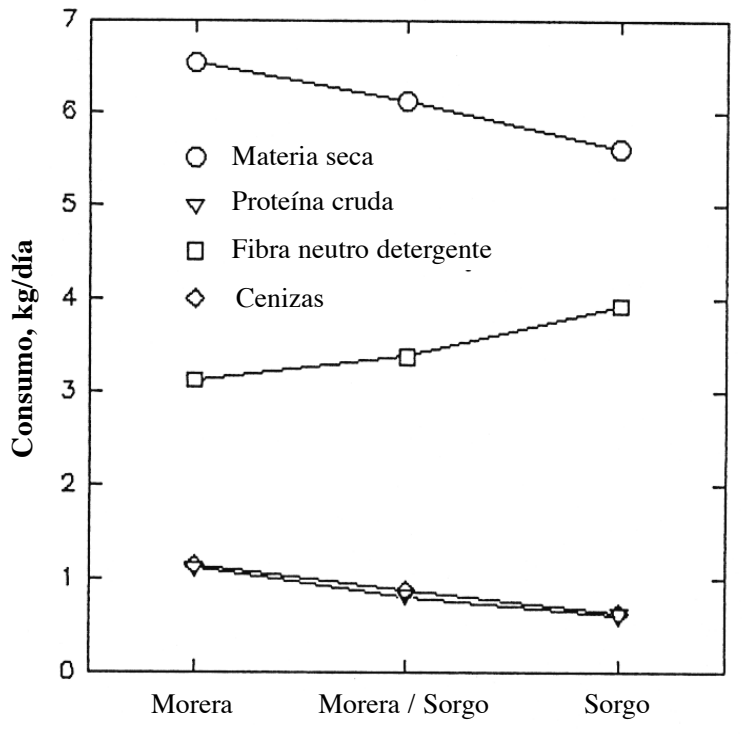

Figura 1. Consumo de nutrimentos de las dietas

lación de 1,94\% para la dieta de morera, 1,82\% para la mezcla morera/sorgo y $1,67 \%$ para la dieta de sorgo negro, lo cual fue inferior al $2 \%$ esperado.

Cuadro 1. Consumo y composición química de las dietas experimentales.

\begin{tabular}{|c|c|c|c|}
\hline Dietas experimentales & Morera & Morera/sorgo & Sorgo negro \\
\hline \multicolumn{4}{|l|}{ Materia fresca } \\
\hline Consumida, kg/día & 24,21 & 24,92 & 24,92 \\
\hline Consumida, $\mathrm{kg} / \mathrm{día} 100 \mathrm{~kg}$ del animal & 7,18 & 7,39 & 7,39 \\
\hline \multicolumn{4}{|l|}{ Materia seca } \\
\hline Ofrecida, $\%$ & $25,81 \mathrm{a}$ & $22,92 \mathrm{~b}$ & $21,40 \mathrm{~b}$ \\
\hline Consumida, $\%$ & $26,62 \mathrm{a}$ & $25,06 \mathrm{a}$ & 24,88 a \\
\hline Consumida, kg/día & $6,53 \mathrm{a}$ & $6,13 \mathrm{a}$ & $5,62 \mathrm{a}$ \\
\hline Consumida, g/día/kg 0,75 del animal & 82,66 & 77,59 & 71,14 \\
\hline Consumida, g/día/100 kg del animal & 1937,69 & 1818,99 & 1667,66 \\
\hline \multicolumn{4}{|l|}{ Proteína cruda } \\
\hline Ofrecida, $\%$ & $13,47 \mathrm{a}$ & $11,17 \mathrm{~b}$ & $10,46 \mathrm{~b}$ \\
\hline Consumida, $\%$ & 17,89 a & $14,04 \mathrm{~b}$ & $11,36 \mathrm{c}$ \\
\hline Consumida, $\mathrm{kg} / \mathrm{día}$ & $1,12 \mathrm{a}$ & $0,82 \mathrm{~b}$ & $0,62 \mathrm{c}$ \\
\hline Consumida, g/día/kg 0,75 del animal & 14,18 & 10,38 & 7,85 \\
\hline Consumida, g/día/100 kg del animal & 332,34 & 243,32 & 183,98 \\
\hline \multicolumn{4}{|l|}{ Fibra neutro detergente } \\
\hline Ofrecida, $\%$ & 56,26 a & $64,24 \mathrm{~b}$ & $72,91 \mathrm{c}$ \\
\hline Consumida, $\%$ & $45,31 \mathrm{a}$ & $53,64 \mathrm{~b}$ & $68,16 \mathrm{c}$ \\
\hline Consumida, $\mathrm{kg}$ /día & $3,12 \mathrm{a}$ & $3,38 \mathrm{a}$ & $3,92 \mathrm{a}$ \\
\hline Consumida, g/día/kg 0,75 del animal & 39,49 & 42,78 & 49,62 \\
\hline Consumida, g/día/100 kg del animal & 925,82 & 1002,97 & 1163,20 \\
\hline \multicolumn{4}{|l|}{ Cenizas totales } \\
\hline Ofrecida, $\%$ & $14,22 \mathrm{a}$ & $12,47 \mathrm{~b}$ & $11,21 \mathrm{c}$ \\
\hline Consumida, $\%$ & $7,67 \mathrm{a}$ & $7,66 \mathrm{a}$ & $7,67 \mathrm{a}$ \\
\hline Consumida, $\mathrm{kg}$ /día & $1,14 \mathrm{a}$ & $0,89 \mathrm{~b}$ & $0,64 \mathrm{c}$ \\
\hline Consumida, g/día/kg 0,75 del animal & 14,43 & 11,27 & 8,10 \\
\hline Consumida, g/día/100 kg del animal & 338,28 & 264,09 & 189,91 \\
\hline
\end{tabular}

a,b,c Diferente letra entre dietas es significativo al $5 \%$. 
Se reconoce que el sorgo negro forrajero tiene una excelente aceptación en el ganado bovino y caprino, ofrecido en forma picada. La morera mostró en el presente experimento, un consumo de materia verde similar a la dieta única de sorgo o en mezcla con sorgo. $\mathrm{Al}$ analizar la concentración de materia seca ofrecida y consumida, se encontró que la materia seca consumida fue siempre superior a la ofrecida, mostrando la selección que hace el animal durante la labor de consumo de alimentos, esto probablemente se debe a la preferencia del animal por las hojas, donde se encuentra el mayor contenido de materia seca en ambos alimentos forrajeros. La dieta ofrecida de morera tuvo un $25,81 \%$ y la consumida un $26,62 \%$ mientras que el sorgo negro ofrecido a los animales contenía $21,40 \%$ y la consumida un $24,88 \%$. Esto refleja que los animales con la dieta de sorgo hicieron un mayor esfuerzo de selección que los animales que recibieron morera. Debe tomarse en cuenta que la morera fue ofrecida con el tallo en estado leñoso. Aún así, la morera tiene una mejor relación hoja:tallo que la encontrada en el sorgo negro. En ambos casos los animales tuvieron el mismo nivel de consumo. La mezcla de morera y sorgo reflejó resultados intermedios. Los niveles de consumo en este experimento no alcanzaron el $2 \%$ esperado. El valor más cercano se obtuvo con la dieta de morera. González, J.G. (1996) evaluando la calidad nutricional de la planta entera de morera en bovinos de engorde (118 a $250 \mathrm{~kg}$ de peso) encontró consumos de materia seca de 0,90, 1,71 y $2,11 \%$ del peso de los animales al ofrecer 1,0, 1,9 y $2,8 \%$ de materia seca de morera en relación al peso vivo. En el mismo orden, las ganancias de peso fueron de 687, 942 y 954 gramos diarios. En forma similar se observó un aumento en el consumo de nutrimentos al incrementar el nivel de morera en la dieta, sustituyendo una dieta de pasto Elefante (Pennisetum purpureum). En vacas lecheras, Oviedo, F.J. (1995), encontró que la producción de leche en vacas disminuyó $400 \mathrm{~g} /$ diarios ( $\mathrm{P}>0,05)$ al sustituir el consumo de materia seca de concentrado (17,6\% de proteína cruda) por hojas de morera ( $18 \%$ de proteína cruda) en la cantidad de $1 \%$ en relación al peso vivo de los animales. Eswara, et al (1991), reportó consumos de materia seca en base al peso metabólico muy inferiores en cabras y similares en ovejas a los observados en el presente estudio con bovinos.

\section{CONCLUSIONES Y RECOMENDACIONES}

La aceptación de la morera y del sorgo forrajero, medida por la cantidad diaria de materia verde consumida fue similar (de 7,18 a 7,38 kg/100 kg de peso vivo). El consumo de materia seca fue ligeramente superior con la dieta de morera $(1,94 \% / 100 \mathrm{~kg})$ al compararse con la mezcla de morera/sorgo $(1,82 \%)$ y con la dieta de sorgo negro $(1,67 \%)$. Este mismo comportamiento se observó en forma más pronunciada, al comparar el consumo diario de proteína cruda y de cenizas totales, debido a la mayor concentración de ambos nutrientes en la morera. El consumo de fibra neutro detergente fue menor en la morera debido a su bajo contenido, cerca de un $23 \%$ menor que en el sorgo negro forrajero.

La adición de morera en la alimentación de animales que consumen dietas basadas en forrajes de gramíneas, ayudará a disminuir la concentración de fibra e incrementará el contenido de proteína y minerales que enriquecen la dieta consumible de los animales. Esto mejorará la cantidad de materia seca consumida diariamente. Con los resultados de este experimento, se recomienda llenar los requerimientos nutricionales tomando la cantidad de materia seca realmente consumible de cada especie forrajera y estimar la cantidad de forraje a ofrecer, considerando la relación de hojas y tallos que componen la dieta completa.

\section{LITERATURA CITADA}

A.O.A.C. 1980. Methods of análisis. Ed. 13 Washingon D.C. EUA. Association of official analysis chemistry.

BENAVIDES, J.; LACHAUX, M.; FUENTES, M. 1994. Efecto de la aplicación de estiércol de cabra en el suelo sobre la calidad y producción de biomasa de morera (Morus sp.). Arboles y arbustos forrajeros en América Central. Serie técnica. CATIE. Informe técnico $N^{\circ} 236$. Volumen II p. 495-514.

BOSCHINI, C.; DORMOND, H.; CASTRO, A. 1998. Producción de biomasa de morera (Morus alba) en la Meseta Central de Costa Rica, establecida y cosechada a diferentes distancias de siembra, alturas, y frecuencias de poda. Agronomía Mesoamericana 9(2):28-39.

CASTRO, A. 1989. producción de leche de cabras alimentadas con King grass (Pennisetum purpureum $\times$ P. typloides), suplementales con diferentes niveles de follaje de poró (Erithina poeppigiana) y de fruto de plátano va de (Musa sp. Var. Pelipita). Tesis MSc Turrialba, C.R. UCR/CATIE. 58p.

CASTRO, A.; BENAVIDES, J. 1994. Evolución de los sistemas de alimentación en explotaciones caprinas de Costa Rica. Arboles y arbustos forrajeros en América Central. Serie técnica CATIE. Informe técnico $\mathrm{N}^{\circ} 236$. Volumen II. p. 245-250.

ESPINOZA, E. 1996. Efecto del sitio y del nivel de fertilización nitrogenada sobre la producción y calidad de tres variedades de morera en Costa rica. Tesis MSc. Centro Agronómico Tropical de Investigación y Enseñanza (CATIE), Turrialba, Costa Rica. 79p. 
ESTRADA, A. 1995. Efecto de la sustitución del King Grass por morera sobre los parámetros de degradación y fermentación ruminal de cuatro forrajes de calidad constante. Tesis MSc., Turrialba, Costa Rica. 60p.

ESWARA, P.; REDDY, M.R. 1992. Nutritive value of Mulberry (Morus alba) leaves in gota and sheep. Indian Journal of Animal Science 8(4):295-296.

GOERING, H.K.; VAN SOEST, P.J. 1970. Forrage fiber analysis (Apparatus, reagents, procedures and some applications). Agricultural handbook $N^{\circ} 379$. ARS-USDA, Washington, D.C. p.

GONZALEZ, J.G. 1996. Evaluación de la calidad nutricional de la morera fresca y ensilada con bovinos de engorde. Tesis MSc. Centro Agronómico Tropical de Investigación y Enseñanza (CATIE), Turrialba, Costa Rica. 65p.

ORTIZ, G. 1992. Efecto de la alimentación con pasto King Grass. (Pennisetum purpureum $\mathrm{x}$ P. typohoides), suplementado con diferentes niveles de follaje de morera (Morus alba) y de banano verde (Musa sp.) sobre la producción de leche de cabra. Tesis Lic. Escuela de zootecnia. Universidad de Costa Rica. 45p.

OVIEDO, F.J. 1995. Morera (Morus alba) en asocio con poró (Erithina poeppigiana) como suplemento para vacas lecheras en pastoreo. Tesis MSc. Centro Agronómico Tropical de Investigación y Enseñanza (CATIE), Turrialba, Costa Rica. 92p.
PICCIONI, M. 1970. Diccionario de alimentación animal. Editorial acribia. Zaragoza. pp. 492-494.

RAO, S.B.N.; NAWAB, S.; OGRA, J.L.; SINGH,N. 1996. Tree leaves based pelleted feeds and their utilization in gotas. Indian Journal of Animal Nutrition. 13(3):174177.

RODRIGUEZ,C.; ARIAS,R.; QUIÑÓNEZ, J. 1994. Efecto de la frecuencia de poda y el nivel de fertilización nitrogenada, sobre el rendimiento y calidad de la biomasa de morera (Morus alba) en el trópico seco de Guatemala. Arboles y arbustos forrajeros en América Central. Serie técnica. CATIE. Informe Técnico $N^{\circ} 236$ Volumen II pp. 515-528.

SUBBARAYAPPA, C.T.; GOWDA, S.K.K.; MUNIYAPPA, T.V.; MANJUNATHA, S. 1995. Effect of different sources of nitrogenous fertilizers on the yield and nutritive value of mulberry (Morus alba Linn). Mysore Journal of Agricultural Sciences 29 (3): 237-243.

SAS. 1985. Statistical analysis system. SAS User's guide: Statistics (versión 5 ed) SAS Institute inc. Cary, NC. p.

YEN, G.C.; WU, S.C.; DUH,P.D. 1996. Extraction and identification of antioxidant components from the leaves of mulberry (Morus alba L.) Journal of Agricultural and Food Chemistry 44 (7): 1687-1690. 